\title{
Dengue Fever: Clinical Characteristics of A Tertiary Care Hospital Study
}

\author{
MOHAMMED RIZWANUL AHSAN ${ }^{1}$, MANZOOR HUSSAIN², SK AZIMUL HOQUE ${ }^{3}$, AL-AMIN MRIDHA ${ }^{4}$, \\ SABRINA MAKBUL ${ }^{5}$
}

\begin{abstract}
:
Background: Incidence of dengue infection has increased worldwide and has become a significant public health concern. Clinical suspicion based on the frequency of symptoms is very important for early diagnosis.

Objectives: To observe the clinical characteristics of serologically confirmed hospitalized cases of dengue fever.

Material and Methods: This cross-sectional study was done among admitted children with dengue infection in Dhaka Shishu (Children) Hospital from July 2018 to October 2018. The diagnosis of dengue fever (DF), dengue hemorrhagic fever (DHF), and dengue shock syndrome (DSS) were established according to the World Health Organization (WHO) classification criteria. Cases who were NS1 antigen and lgM dengue antibody positive included in this study.

Results: Among 51 serologically confirmed dengue fever patients, mean age was $6.66 \pm 3.69$ years. Majority of the cases $(74.5 \%)$ were less than 10 years of age, $60.8 \%$ were male, $39.2 \%$ were female, and $88.23 \%$ of patients came from urban areas. Classic DF was found in $74.5 \%$ of patients, while $25.49 \%$ of patients DHF, and $11.7 \%$ DSS. Fever was the most common (72.55\%) symptom followed by headache, vomiting and myalgia. Hemorrhagic manifestations found in $21.57 \%$ of cases. The most common complications were hepatic dysfunction (47.09\%) followed by renal impairment, encephalopathy, multi-organ failure, and ARDS.

Conclusion: The majorities of dengue cases were from urban areas, below ten years of age and classical DF. Besides fever other common symptoms were headache, vomiting and myalgia. The most common complications were hepatic dysfunction, renal impairment and encephalopathy.
\end{abstract}

Keywords: Dengue fever (DF), Dengue Shock Syndrome (DSS), Dengue Hemorrhagic Fever (DHF).

1. Assistant Prof. Department of Adolescent Medicine \& Resident Physician Dhaka Shishu (Children) Hospital, Dhaka.

2. Ex-Director, Dhaka Shishu (Children) Hospital, Dhaka.

3. Associate Professor, Department of Paediatric Neurology NINS, Dhaka, Bangladesh

4. Professor of Paediatrics, Department of Paediatrics, Shaheed Suhrawardy Medical College \& Hospital, Dhaka, Bangladesh

5. Senior Nutritionist, Dhaka Shishu (Children) Hospital, Dhaka, Bangladesh

Correspondence: Dr. Mohammed Rizwanul Ahsan, Assistant Prof, Depart of Adolescent Medicine \& Resident Physician Dhaka Shishu (Children) Hospital, Dhaka Bangladesh.

Cell no-8801711465405 Email:rizwanul.ahsan@gmail.com.

Received: $1 / 07 / 2019$

Accepted: 15/03/2020

\section{Introduction}

Around the world incidence of dengue infection has increased and in recent decades and has become a major international public health concern. Worldwide around 2.5 billion people continue to live at risk of contracting dengue infection and yearly 24,000 deaths tend to occur in 100 endemic countries. ${ }^{1}$

In Bangladesh, epidemics are becoming more frequent. Increase in the frequency of epidemics and involvement of younger age group are indicators of higher incidence of infection. 2,3 Dengue was first reported in 1780, when Benjamin Rush described 
this condition as "break bone fever". This mosquitoborne viral infection caused by one of the four serotypes of the dengue virus (DENV; (DENV-1 to DENV-4) belonging to the family Flaviviridae. These serotypes are closely related but antigenically distinct. Wide spectrum of disease severity ranging from an influenza-like illness-dengue fever (DF) to the lifethreatening dengue hemorrhagic fever (DHF)/dengue shock syndrome (DSS) may happen from dengue infection and are associated with mortality as high as $20 \%$ if untreated. ${ }^{4-6}$ Early diagnosis is essential and clinical suspicion is based on the frequency of symptoms in the population. Though it was common in Africa, the first confirmed report of dengue infection in Bangladesh dates back to the 1960s. Since then more and more newer parts of the world have been reporting the disease which mostly strikes in epidemic with high morbidity and mortality. ${ }^{7}$ Several fatal forms of the disease i.e., DHF, DSS has been reported from various parts of Bangladesh. In different parts of the world, children were affected in all those epidemics though most of the cases were from 16-60 year age group. ${ }^{8,9}$ The common signs and symptoms observed were fever, headache, myalgia, arthralgia, and bleeding manifestations. The exact clinical profile is important for better management and prognosis. The objectives of the study were to assess the clinical and laboratory profile of serologically proven DF in a tertiary care hospital.

\section{Materials and Methods}

A hospital-based cross-sectional study was undertaken in the department of paediatrics, Dhaka Shishu (Children) Hospital from July 2018 to October 2018. A total of 51 children with confirmed dengue infection who fulfilled the WHO classification criteria $^{2}$ of DF, DHF, and DSS were enrolled in this study. Children admitted with other types of infective disease (e.g. malaria, Enteric fever, meningitis, urinary tract infection, respiratory tract infection), any prior history of bleeding tendency, and immunocompromised patients were excluded from this study.

Detailed history and clinical examinations were done. Hematological profiles ( $\mathrm{Hb} \%, \mathrm{TC}, \mathrm{DC}$ of WBC, Platelet count, Hematocrit) and other biochemical investigations (e.g. ALT, S. creatinine, S. electrolytes, S. glucose) were assessed at the time of admission. Evidence of plasma leakage was assessed by chest radiograph and abdominal ultrasonography. NS1 antigen and $\operatorname{lgM}$ and $\lg \mathrm{G}$ dengue antibody were measured by using ELISA in all suspected cases. Finally, 51 dengue cases out of 101 were confirmed by either NS1 or IgM dengue antibody positivity. Clinical and laboratory data were collected and then analyzed. Informed written consent was taken from parents, and ethical clearance from the ethical committee of Dhaka Shishu Hospital was also taken before enrollment in the study.

Statistical analysis was performed by the Chi-Square test by using the Statistical Package "SPPS" for Social Sciences, with $p<0.05$ taken as statistically significant.

\section{Results}

Among the total of 101 suspected cases, 51(50.49\%) cases had serologically confirmed dengue infection. The mean age in this study population was $6.66 \pm$ 3.69 years (ranging 1-15 year). Maximum number of cases $38(74.5 \%)$ were from below 10 year age group followed by $13(25 \%)$ cases within $10-15$ years. (Table-I). Male were predominant 31(60.8\%) and $20(39.2 \%)$ were female. Most of the cases were from urban $45 / 51$ (88.23\%) and only 6/51 (11.77\%) from rural area. Considering the clinical type of dengue infection majority $38(74.51 \%)$ cases were classic dengue fever, and DHF were $13(25.49 \%)$ cases. Among the 13 cases of DHF, six patients developed DSS (Table II). In this study, most common symptoms were fever $(72.55 \%)$, followed by headache (52.94\%), and vomiting (41.18\%). Different types of hemorrhagic manifestations were found in $11(21.57 \%)$ cases, which include petechiae, ecchymosis, gum bleeding, hematuria, melena, hematemesis, and epistaxis. Most common complications were hepatic dysfunction $(47.09 \%)$, renal failure $(37.25 \%)$, multi-organ failure $(9.80 \%)$, encephalopathy (15.68\%) and ARDS (3.92\%).

Table I

Age distribution of patients with Dengue fever $(n=51)$.

\begin{tabular}{lccc}
\hline Age (Years) & Male & Female & Total $(\%)$ \\
\hline 1-5 Yrs. & 12 & 07 & $19(37.25)$ \\
5-10 Yrs. & 11 & 08 & $19(37.25)$ \\
10-15 Yrs. & 08 & 05 & $13(25.50)$ \\
Total & $31(60.8 \%)$ & $20(39.2 \%)$ & 51 \\
\hline
\end{tabular}

Mean age of $6.66 \pm 3.69$ years

Range 1 year to 15 years 
BANGLADESH J CHILD HEALTH 2020; VOL 44 (1) : 32 Dengue Fever: Clinical Characteristics of A Tertiary Care Hospital Study

Table -II

Showing the frequency of different type of dengue fever $(n=51)$

\begin{tabular}{lcc}
\hline Classification & Number & Percent \\
\hline Classic DF & 38 & $(74.51 \%)$ \\
DHF & 13 & $(25.49 \%)$ \\
DSS & 6 & $(11.76)$ \\
\hline
\end{tabular}

\section{Table III}

Symptoms and complications of dengue fever (multiple symptoms were found in most cases) $(n=51)$.

\begin{tabular}{lc}
\hline Symptoms & Patients (\%) \\
\hline Fever & $37(72.55)$ \\
Headache & $27(52.94)$ \\
Vomiting & $21(41.18)$ \\
Myalgia & $19(37.25)$ \\
Breathlessness & $15(29.41)$ \\
Abdominal pain & $13(25.49)$ \\
Bleeding tendency & $11(21.57)$ \\
Skin rash & $7(13.73)$ \\
Complications & \\
Hepatic dysfunction & $24(47.06)$ \\
Renal failure & $19(37.25)$ \\
Encephalopathy & $8(15.69)$ \\
Multi organ failure & $5(9.80)$ \\
ARDS & $2(3.92)$ \\
\hline
\end{tabular}

Among 51 confirmed dengue cases, 39 (76.5\%) cases were NS1 antigen positive, and 12 (73.5\%) cases IgM antibodies and 8(20.6\%) were both IgM and IgG antibody positive. (Table IV).

Table IV

Distribution of the serologically dengue positive patients $(n=51)$.

\begin{tabular}{lcc}
\hline Serological test & Positive & Percentage \\
\hline NS1 Ag & 39 & 76.5 \\
IgM & 12 & 23.5 \\
Both IgM and IgG & 8 & 20.6 \\
\hline
\end{tabular}

\section{Discussion}

Dengue is a significant emerging disease and can present with varied manifestations. ${ }^{9,10}$ Male predominance was found in this study, which is similar to the study of Chandralekha et al. ${ }^{11}$ In this study, the majority of the child was below 10 year age group. Reddy et al ${ }^{12}$ also found mean age of affected child $8.3 \pm 3.02$ years, which is similar to our findings. Considering presenting symptoms next to fever (72.55\%), headache $(52.94 \%)$, vomiting $(41.18 \%)$, myalgia (37.25\%) and hemorrhagic manifestations $(21.57 \%)$ were commonly found in this study. Reddy GC et al. ${ }^{12}$ in their study found fever as most common symptom, followed by skin rash $(56.0 \%)$, myalgia $(52.0 \%)$ and head ache $(48.0 \%)$ were the symptoms which is little bit different from this study but our findings were similar to study of Kumar $A$ et al. ${ }^{13}$ Abdominal pain and vomiting were also common presenting symptom in this study which may often lead to a delay in the diagnosis of dengue. ${ }^{14}$ Deranged liver operate in dengue fever infection is a result of the direct impact of the virus on liver cells or the unregulated host response against the virus may cause fulminant hepatic failure from acute hepatitis and massive necrosis of the liver, causing hepatic encephalopathy and even death. ${ }^{15,16} \mathrm{We}$ also found hepatic dysfunction as the most common complication $(47.06 \%)$ in this study. Shah I et al ${ }^{17}$ reported altered sensorium (58\%), diarrhoea (50\%), rash $(42 \%)$, and cough $(38 \%)$ in a significant number of cases and headache was seen less often which can be explained more cases of DHF and DSS in their study. Among the cases with dengue infection most of the children had classical dengue fever while DHF and DSS were less in number in our study, which is similar to the Indian study of Batra $\mathrm{T}$ et $\mathrm{al}^{18}$. Hemorrhagic manifestations was found in $21.57 \%$ cases in dengue infection in this study and that were in different form included petechiae, ecchymosis, gum bleeding, hematuria, melena, hematemesis and epistaxis. Large number of cases developed complications and most common complications were hepatic dysfunction, renal impairment and encephalopathy which is similar to other studies. ${ }^{11,13}$ Early clinical features of dengue infection are variable and often non-specific ${ }^{2,19}$ Increase in the number of dengue cases may be due to the rapid urbanization. ${ }^{20}$

\section{Conclusion}

The majority of dengue cases were from urban areas and below 10 years. In dengue infection besides fever 
other common symptoms were headache, vomiting and myalgia. The most common complications were hepatic dysfunction, renal impairment and encephalopathy.

\section{References}

1. World health Organization. Dengue and dengue hemorrhagic fever. Fact Sheet. No. 117,2002. Availablefrom:http//www.who.int/mediacentre/ factsheet s/fs117/en/.

2. World Health Organization. Dengue Hemorrhagic Fever: Diagnosis, Treatment, Prevention and Control. 2nd edition. Geneva, World Health Organization, 1997. Available from https:// www.who.int/csr/resources/publications/dengue/ Denguepublication/en/.

3. World Health Organization. WHO report on global surveillance of Epidemic prone infectiousdiseases? http://www/who.int/emcdocuments/surveillance/ docs/whocdscsrisr 2001.html

4. WHO Fact Sheet $N^{\circ}$ 117: Dengue and dengue hemorrhagic fever. 2008. Available from: http:// www.who.int/mediacentre/factsheets/fs117/en=.

5. Dengue and Dengue Hemorrhagic Fever: Information for Health Care Practitioners - CDC Division of Vector-Borne Infectious Diseases. Available from: http://www.cdc.gov/ncidod/dvbid/ dengue/dengue-hcp.htm.

6. WHO (2009) Dengue Guidelines for Diagnosis, Treatment, Prevention and ControlWHO(2009)http:/ /whqlibdoc.who.int/publications/2009/978924 1547871 _eng.pdf.

7. Dengue in Kerala: A critical review. ICMR Bulletin. 2006; 36:13-22.

8. Balaya S, Paul SD, D'Lima LV, Pavri KM. Investigations on an outbreak of dengue in Delhi in 1967. Indian J Med Res. 1969; 5:767- 74.

9. Chaturvedi UC, Mathur A, Kapoor AK, Mehrotra NK, Mehrotra RM. Virological study of an epidemic of febrile illness with hemorrhagic manifestations at Kanpur, India during 1968. Bull World $\mathrm{H}$ e a I t h Organ.1970; 4:289-93.
10. Prabhakar $\mathrm{H}$, Mathew $\mathrm{P}$, Marshalla $\mathrm{R}$, Arya M. Dengue hemorrhagic fever outbreak in OctoberNovember 1996 in Ludhiana, Punjab, India. Indian J Med Res. 1997; 106:1-3.

11. Chandralekha, Gupta P, Trikha A. The north Indian dengue outbreak 2006: a retrospective analysis of intensive care units admissions in a tertiary care hospital. Trans R Soc Trop Med Hyg. 2008;102: 143-47.

12. Reddy GC, Nagendra K. Clinical and diagnostic features of dengue haemorrhagic fever in children. Int J Contemp Pediatr. 2018;5: 791-97.

13. Kumar A, Rao CR, Pandit V, Shetty S, Bammigatti $\mathrm{C}$, Samarasinghe CM. Clinical Manifestations and Trend of Dengue Cases Admitted in a Tertiary Care Hospital, Udupi, Karnataka. Indian J Community Med. 2010; 35 : 386-90.

14. Khan E, Siddiqui J, Shakoor S, Mehraj V, Jamil B, Hasan R. Dengue outbreak in Karachi, Pakistan, 2006: experience at a tertiary care center. Trans $R$ Soc Trop Med Hyg. 2007;101: 1114-19.

15. Martina BE, Koraka P, Osterhaus AD. Dengue virus pathogenesis: an integrated view. Clin Microbiol Rev. 2009;22: 564-81.

16. Ritu Karoli, Jalees Fatima, Zeba Siddiqi, Khursheed I. Kazmi, Amit R. Sultania. Clinical profile of dengue infection at a teaching hospital in North India. J Infect Dev Ctries. 2012; 6: 551-54.

17. Shah I, Deshpande GC, Tardeja PN. Outbreak of Dengue in Mumbai and Predictive Markers of dengue Shock Syndrome. J Trop Pediatr. 2004;50: 301-305.

18. Batra P, Saha A, Chaturvedi P, Vilhekar KY, Mendiratta DK. Outbreak of dengue infection in rural Maharashtra. Indian J Pediatr. 2007;74: 794-95.

19. De Oliveira SA, Bastos Camacho LA, Fernandes Bruno L, de Gusmão RC, de Medeiros Pereira AC, Coca Velarde LG, et al. Acute arthropathy in patients with rash diseases: a comparative study. Clin Rheumatol. 2009;28: 1067-71.

20. Gubler DJ. Dengue and dengue hemorrhagic fever. Clin Microbiol Rev. 1998;11: 480-96. 